\title{
SELECTIVE WITHDRAWAL OF A TWO-LAYER VISCOUS FLUID
}

\author{
JASON M. COSGROVE ${ }^{\otimes 1}$ and LAWRENCE K. FORBES ${ }^{1}$
}

(Received 15 December, 2011; revised 3 October, 2012; first published online 8 February, 2013)

\begin{abstract}
Selective withdrawal of a two-layer fluid is considered. The fluid layers are weakly compressible, miscible and viscous and therefore flow rotationally. The lower, denser fluid flows with constant velocity out through one or more drain holes in the bottom of a rectangular tank. The drain is opened impulsively and the subsequent draw-down produces waves in the interface which travel outward to the edges of the tank and are reflected back with a $180^{\circ}$ change of phase. The points on the interface that have the highest absolute gradient form regions of high vorticity in the tank, enabling mixing of the fluids. An inviscid linearized interface is computed and compared to contour plots of density for the viscous solution. The two agree closely at early times in the withdrawal process, but as time increases, nonlinear and viscous effects take over. The time at which the lighter fluid starts to flow out of the tank is dependent on the number of drains, their width, and the fluid flow rate and density, and is investigated here.
\end{abstract}

2010 Mathematics subject classification: primary 76D50; secondary 76M22.

Keywords and phrases: selective withdrawal, multiple drains, viscous fluids, spectral methods, nonlinear effects, two-layered fluids.

\section{Introduction}

There are many instances in nature where two fluid layers of different densities overlay each other. These include oil on water, subterranean layers of fresh and salty water, and lakes that are thermally stratified. These situations also arise in industry. For example, cooling ponds used in power stations have a pronounced stratification in the fluid, where the recently used warmer water lies above the denser cooler water. Due to these artificial and naturally occurring events, a lot of work has been done on the withdrawal of fluid from a two-layered reservoir, particularly in the past few decades [2, 4, 5, 8]. When outflow from a two-layered reservoir is less than some critical value, fluid exits from the layer that contains the sink. This phenomenon is termed selective withdrawal.

\footnotetext{
${ }^{1}$ School of Mathematics and Physics, University of Tasmania, Private Bag 37, Hobart, Tasmania 7001, Australia; e-mail: Jason.Cosgrove@utas.edu.au, Larry.Forbes@utas.edu.au.

(C) Australian Mathematical Society 2013, Serial-fee code 1446-1811/2013 \$16.00
} 
With the increased need to maintain environmental standards worldwide, the problem of extracting fluid from reservoirs consisting of two different density layers has become increasingly important, and thus there are substantial engineering applications in this area. Maintaining water quality and reservoir management as discussed by Imberger and Hamblin [7] are examples, along with the ability to reuse water in industrial settling tanks and withdrawing the coolest water from power station cooling ponds [12]. The better the understanding of the flow pattern as the fluid is being withdrawn, the more efficient and environmentally friendly these industrial applications may become.

Although the literature on selective withdrawal is extensive, the vast majority of the mathematical modelling of such withdrawal problems assumes that the outflow involves only a single sink, the fluids are inviscid, immiscible and incompressible, and the flow is irrotational. This was assumed by Forbes and Hocking [4] and Hocking [5]. Forbes and Hocking [4] showed that for an impulsive start in the draining process, the nonlinear and linearized interfaces between the fluids coincide for early times, but as the interface draws closer to the drain, the nonlinear and linearized solutions vary considerably as nonlinear effects start to dominate. They also found that curvature singularities apparently develop on the interface, for wide drains. As fluid is being withdrawn, waves are produced in the interfacial region of the fluid system. Depending on the outflow rate, these waves can have varying amplitudes and therefore their influence on the withdrawal is significant. Farrow and Hocking [2] showed that this behaviour can delay or accelerate the arrival of the interface at the sink. Ahmed et al. [1] consider withdrawal from multiple holes, although these are arranged vertically, and so that problem is largely equivalent to selective withdrawal through a single sink in a horizontal bottom.

The viscous problem is much more difficult since it involves the Navier-Stokes equations. Additionally, the conditions on the interface or free surface are much more complicated. Viscous effects have had relatively little consideration in the literature. Nevertheless, Imberger et al. [8] looked at selective withdrawal from a finite rectangular tank, taking viscosity into account, with a sink located at the bottom left corner. They showed that the natural processes of the withdrawing layer depend on viscous forces, the number of reflections of the wave off the wall opposite the sink, and the Prandtl number. Lister [9] considered withdrawal of a two-layered viscous fluid through a point sink located centrally in a semi-infinite cylinder, under the approximation that the nonlinear inertial terms in the equations of motion were negligible. More recently, Hocking and Zhang [6] also looked at viscous fluids and considered withdrawal from a two-layered fluid through a line sink in a porous medium. In this case, the line sink is situated above an impermeable wedge-shaped region. The results can be used to improve withdrawal of viscous fluids from oil reserves and ground water aquifers.

Farrow and Hocking [2] approximated the two-layer fluid system with a single fluid of spatially varying density and used Boussinesq approximation theory. A finite 
difference method was used to solve the problem for a single hole located at the bottom left corner of the tank, with a range of values for the physical parameters.

In this paper, we consider the withdrawal of fluid from a two-layer fluid system confined to a rectangular tank. We follow Farrow and Hocking [2] and also use Boussinesq approximation theory. However, we focus on designing a tank with multiple drain holes, not necessarily symmetrically placed, to achieve maximum withdrawal volume before the interface reaches the sink or sinks. The fluids are weakly compressible, viscous and miscible, and flow rotationally. Density and vorticity contour plots for tanks with various drain widths and flow rates are of particular interest, together with different drain configurations. These nonlinear viscous solutions are obtained using a novel spectral method, and are compared with linearized approximations to the inviscid problem. Nonlinear and viscous effects are seen at later times in the withdrawal process.

\section{Mathematical formulation}

We consider a two-dimensional rectangular tank that lies in the $x y$-plane. The $x$ axis is located along the bottom of the tank, with the $y$-axis oriented vertically upward from the centre of the tank. The left side wall is located at $x=-W$ and the right side wall at $x=W$. This results in a tank of width $2 W$. The bottom of the tank has a drain hole located centrally with width $2 A$, so that the left and right sides of the drain hole are located at $x=-A$ and $x=A$, respectively, on $y=0$.

The tank contains two different density fluids that have the same viscosity coefficient, with the denser fluid below the lighter fluid. The initial interface between the two fluids is horizontal and located at $y=H$. The lighter fluid, located in region 1 of the tank, is considered to have density $\rho_{1}$ and thickness $L$. The denser fluid in region 2 has height $H$ and density $\rho_{2}$. It follows that $\rho_{1}<\rho_{2}$. The tank has height $y=H+L$.

At time $t=0$ the drain is turned on impulsively. The withdrawal speed at the drain is $V_{0}$, and the fluid is subject to the acceleration due to gravity $g$. It is assumed that constant outflow occurs over the region of the drain hole, giving an outward mass flux $2 A V_{0}$. It is required that the tank be full at all times, and it must therefore be recharged uniformly at the top with the same volume of fluid that is being drained out. This leads to the inward mass flux of $2 A V_{0} / W$ injected at the top of the tank.

The fluid is assumed to be weakly compressible, and accordingly the interface between the fluid regions can be regarded as a rapid but smooth transition from one density to another. The density $\rho$ can then be expressed as $\rho=\rho_{1}+\bar{\rho}$, where $\rho_{1}$ is the density of the upper, lighter fluid and $\bar{\rho}$ is a small perturbation to it.

Dimensionless variables are introduced immediately, and are used throughout this paper. All lengths are scaled relative to $H$, the initial depth of the lower, heavier fluid and the time scale is taken to be $\sqrt{H / g}$. The density scale is chosen to be the lower fluid density $\rho_{2}$ and the speed scale is $\sqrt{g H}$. The problem is then dependent on seven 


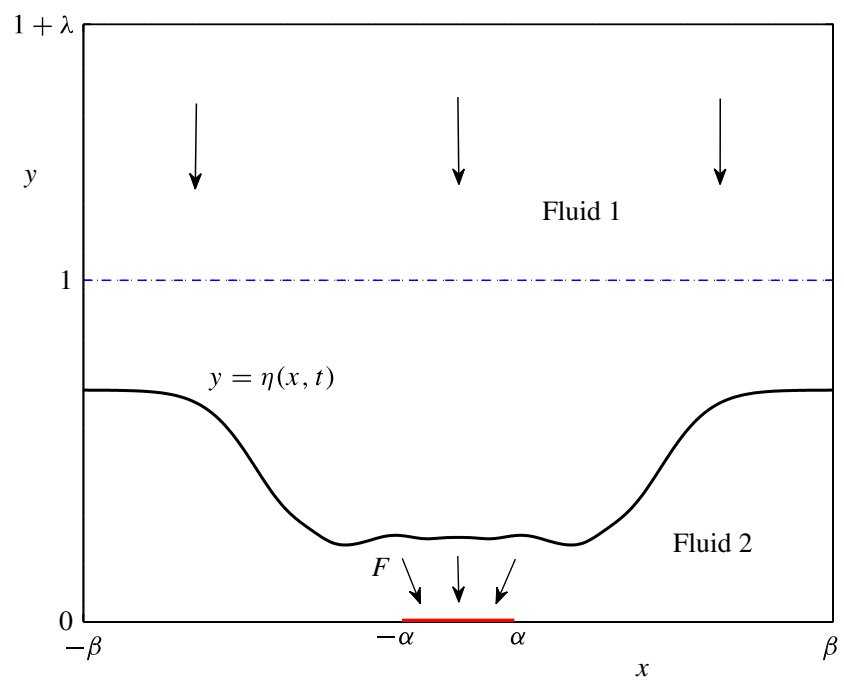

FIGURE 1. Sketch of the nondimensionalized tank. Initially, the interface is at height $y=1$ (dashed line), but drops as time progresses. The interface (solid line) has been taken from an inviscid linearized solution with $F=0.05$ and $D=0.95$ at time $t=75$.

dimensionless constant parameters:

$$
\begin{aligned}
& \alpha=\frac{A}{H}, \quad \beta=\frac{W}{H}, \quad \lambda=\frac{L}{H}, \quad D=\frac{\rho_{1}}{\rho_{2}}, \\
& \mu=\frac{v}{\sqrt{g H^{3}}}, \quad F=\frac{V_{0}}{\sqrt{g H}}, \quad \gamma=\frac{\sigma}{\sqrt{g H^{3}}} .
\end{aligned}
$$

The first constant $\alpha$ in (2.1) represents the dimensionless half-width of the drain, and the second constant $\beta$ is the half-tank width. The constant $\lambda$ represents the dimensionless height of the upper, lighter fluid. Consequently, in dimensionless variables the initial height of the lower, heavier fluid is 1 and the total height of the tank is $1+\lambda$. The width of the tank is $2 \beta$ and the drain width is $2 \alpha$. A sketch of the nondimensionalized tank is given in Figure 1. The parameter $D$ is the density ratio between the upper and lower fluids. The parameter $\mu$ is the dimensionless viscosity coefficient and may be thought of as the inverse of a Reynolds number. The parameter $F$ is a Froude number based on the initial depth of the lower, heavier fluid and can be considered as a measure of the flow rate out through the drain hole. The parameters $\sigma$ and $\gamma$ are the dimensional and nondimensional diffusion coefficients, respectively.

The velocity vector is written $\mathbf{q}=u \underline{i}+v \underline{j}$ where $u$ and $v$ denote the horizontal and vertical velocity components, respectively. In Boussinesq theory, the mass conservation equation is split into an essentially incompressible part

$$
\frac{\partial u}{\partial x}+\frac{\partial v}{\partial y}=0
$$


and an effective transport component for the perturbation density, which takes the form

$$
\frac{\partial \bar{\rho}}{\partial t}+\mathbf{q} \cdot \nabla \bar{\rho}=\gamma \nabla^{2} \bar{\rho}
$$

The viscous momentum equations for the horizontal and vertical velocity components, respectively, are

$$
\begin{aligned}
& \frac{\partial u}{\partial t}+\mathbf{q} \cdot \nabla u+\frac{\partial p}{\partial x}=\mu \nabla^{2} u \\
& \frac{\partial v}{\partial t}+\mathbf{q} \cdot \nabla v+\frac{\partial p}{\partial y}=-\bar{\rho}+\mu \nabla^{2} v
\end{aligned}
$$

Equations (2.4) and (2.5) are combined by differentiating with respect to $y$ and $x$, respectively, and subtracting to eliminate pressure, resulting in the single scalar equation

$$
\frac{\partial \omega}{\partial t}+\mathbf{q} \cdot \nabla \omega=-\frac{\partial \bar{\rho}}{\partial x}+\mu \nabla^{2} \omega
$$

for the vorticity

$$
\omega=\frac{\partial v}{\partial x}-\frac{\partial u}{\partial y}
$$

Equation (2.2) is satisfied identically using the stream function $\Psi(x, y, t)$, in terms of which the velocity components and vorticity become

$$
u=\frac{\partial \Psi}{\partial y}, \quad v=-\frac{\partial \Psi}{\partial x}, \quad \omega=-\nabla^{2} \Psi .
$$

When modelling viscous fluid behaviour it is necessary to enforce a nonslip condition on the walls of the tank, creating a boundary layer. This layer will be extremely thin and its influence in the centre of the tank where the drain holes are located will be negligible. Consequently, it is assumed here that the boundary layers on the two walls and the bottom of the tank can be ignored. We therefore follow Farrow and Hocking [2] in allowing slip boundary conditions on the walls and bottom of the tank. The approximate boundary conditions on the side walls are

$$
u=\omega=0 \quad \text { at } x= \pm \beta,
$$

and on the bottom of the tank at $y=0$,

$$
v= \begin{cases}-F & \text { if }-\alpha<x<\alpha \\ 0 & \text { otherwise }\end{cases}
$$

At the top of the tank, at $y=1+\lambda$, the recharge rate is

$$
v=-\frac{\alpha F}{\beta} \text {. }
$$




\section{The spectral solution method}

The stream function $\Psi$ in equation (2.7) is written as the sum of a steady background component $\Psi_{b}$ and a fully unsteady component $\Psi_{u}$ in the form

$$
\Psi(x, y, t)=\Psi_{b}(x, y)+\Psi_{u}(x, y, t) .
$$

3.1. Single hole located symmetrically The background component $\Psi_{b}$ is determined by ignoring viscosity and looking for steady flow of a single fluid through the tank, to conserve mass. Therefore, $\Psi_{b}$ is the solution to Laplace's equation in the two-dimensional region $-\beta<x<\beta$ and $0<y<1+\lambda$, satisfying boundary conditions $u=0$ on the walls $x= \pm \beta$, the top condition (2.10) on $y=1+\lambda$ and the bottom condition (2.9) on $y=0$. The background component of the stream function can be represented in the form

$$
\Psi_{b}(x, y)=\frac{\alpha F x}{\beta}+\sum_{n=1}^{\infty} B_{n} \sinh \left[\frac{n \pi}{\beta}(1+\lambda-y)\right] \sin \left(\frac{n \pi x}{\beta}\right),
$$

where

$$
B_{n}=\frac{2 \beta F \sin \left(\frac{n \pi \alpha}{\beta}\right)}{n^{2} \pi^{2} \sinh \left[\frac{n \pi}{\beta}(1+\lambda)\right]} .
$$

Since the steady component $\Psi_{b}$ already satisfies the bottom boundary condition (2.9), it is appropriate to take the unsteady component in the form

$$
\Psi_{u}(x, y, t)=\sum_{m=1}^{\infty} \sum_{n=1}^{\infty} A_{m n}(t) \sin \left(\frac{m \pi y}{1+\lambda}\right) \sin \left(\frac{n \pi x}{\beta}\right)
$$

with coefficient functions $A_{m n}(t)$ to be determined. Thus the full representation of the stream function is given by

$$
\begin{gathered}
\Psi(x, y, t)=\frac{\alpha F x}{\beta}+\sum_{n=1}^{\infty} B_{n} \sinh \left[\frac{n \pi}{\beta}(1+\lambda-y)\right] \sin \left(\frac{n \pi x}{\beta}\right) \\
+\sum_{m=1}^{\infty} \sum_{n=1}^{\infty} A_{m n}(t) \sin \left(\frac{m \pi y}{1+\lambda}\right) \sin \left(\frac{n \pi x}{\beta}\right) .
\end{gathered}
$$

Equation (2.7) gives the expression for the vorticity $\omega$ of the fluid in the tank in the form

$$
\omega=\sum_{m=1}^{\infty} \sum_{n=1}^{\infty} C_{m n} A_{m n}(t) \sin \left(\frac{m \pi y}{1+\lambda}\right) \sin \left(\frac{n \pi x}{\beta}\right),
$$

where

$$
C_{m n}=\left(\frac{n \pi}{\beta}\right)^{2}+\left(\frac{m \pi}{1+\lambda}\right)^{2}
$$


The vorticity equation (2.6) shows that the density derivative $\partial \bar{\rho} / \partial x$ must have a similar form to that in equation (3.4) for the vorticity. In addition, it is necessary to make $\bar{\rho}=0$ at the top $y=1+\lambda$ of the tank, and to have $\bar{\rho}=1-D$ at the bottom $y=0$. It is therefore appropriate to take the series for the density perturbation to be

$$
\begin{aligned}
\bar{\rho}(x, y, t)=\sum_{m=1}^{\infty} & \sum_{n=1}^{\infty} B_{m n}(t) \sin \left(\frac{m \pi y}{1+\lambda}\right) \cos \left(\frac{n \pi x}{\beta}\right) \\
& +\sum_{m=1}^{\infty} B_{m 0}(t) \cos \left(\frac{(2 m-1) \pi y}{2(1+\lambda)}\right) .
\end{aligned}
$$

The vorticity equation (2.6) and the density equation (2.3) are decomposed spectrally, by multiplying by basis functions and integrating over the region of the tank. This gives the following system of equations for the time-dependent Fourier coefficients:

$$
\begin{aligned}
A_{k l}^{\prime}(t)= & \frac{-2}{\beta(1+\lambda) C_{k l}} \int_{0}^{1+\lambda} \int_{-\beta}^{\beta} F(x, y, t) \sin \left(\frac{l \pi x}{\beta}\right) \sin \left(\frac{k \pi y}{1+\lambda}\right) d x d y \\
& +\frac{l \pi}{\beta} \frac{B_{k l}(t)}{C_{k l}}-\mu C_{k l} A_{k l}(t), \quad k=1,2, \ldots, M, l=1,2, \ldots, N \\
B_{k l}^{\prime}(t)= & \frac{-2}{\beta(1+\lambda)} \int_{0}^{1+\lambda} \int_{-\beta}^{\beta} G(x, y, t) \cos \left(\frac{l \pi x}{\beta}\right) \sin \left(\frac{k \pi y}{1+\lambda}\right) d x d y \\
& -\gamma C_{k l} B_{k l}(t), \quad k=1,2, \ldots, M, l=1,2, \ldots, N \\
B_{k 0}^{\prime}(t)= & \frac{-1}{\beta(1+\lambda)} \int_{0}^{1+\lambda} \int_{-\beta}^{\beta} G(x, y, t) \cos \left(\frac{(2 k-1) \pi y}{2(1+\lambda)}\right) d x d y \\
& \quad-\gamma\left(\frac{(2 k-1) \pi}{2(1+\lambda)}\right)^{2} B_{k 0}(t), \quad k=1,2, \ldots, M .
\end{aligned}
$$

The constants $C_{k l}$ are given by equation (3.5), and the functions in the integrands are defined for convenience to be

$$
F(x, y, t)=u \frac{\partial \omega}{\partial x}+v \frac{\partial \omega}{\partial y} \quad \text { and } \quad G(x, y, t)=u \frac{\partial \bar{\rho}}{\partial x}+v \frac{\partial \bar{\rho}}{\partial y} .
$$

Equations (3.7)-(3.9) embody a system of $M(1+2 N)$ ordinary differential equations for the time-dependent Fourier coefficients in the representations of the stream function (3.3), the vorticity (3.4) and the perturbation density (3.6). This large system of differential equations is then solved using a fourth-order Runge-Kutta method to integrate the coefficients forward in time.

3.2. Multiple holes located symmetrically In this section, a tank with multiple drain holes is investigated. As previously, the tank has width $2 \beta$ and height $1+\lambda$. The drain holes are centred about $\pm \beta / 3$ and have width $\alpha$. Thus, the left drain hole is located in the region $-\beta / 3-\alpha / 2<x<-\beta / 3+\alpha / 2$, and the right drain hole 
in $\beta / 3-\alpha / 2<x<\beta / 3+\alpha / 2$. The boundary conditions on the walls and top are the same as for the case of a single hole located symmetrically and are given in (2.8) and (2.10). Therefore. the equations representing the stream function (3.3), the vorticity (3.4) and the perturbation density (3.6) remain the same, but the Fourier coefficients $B_{n}$ differ due to the imposed boundary condition at the bottom of the tank changing from (2.9) to

$$
v(x, 0)= \begin{cases}-F & \text { if }|x+\beta / 3|<\alpha / 2 \text { or }|x-\beta / 3|<\alpha / 2 \\ 0 & \text { otherwise }\end{cases}
$$

The Fourier coefficients in equation (3.3) take the form

$$
B_{n}=\frac{2 \beta F\left[\sin \left(\frac{n \pi(-\beta / 3+\alpha / 2)}{\beta}\right)+\sin \left(\frac{n \pi(\beta / 3+\alpha / 2)}{\beta}\right)\right]}{n^{2} \pi^{2} \sinh \left[\frac{n \pi}{\beta}(1+\lambda)\right]} .
$$

3.3. Multiple holes located asymmetrically For convenience, the walls in this case are taken to be located at $x=0$ and $x=2 \beta$, and a virtual tank is to have walls located at $x=-2 \beta$ and $x=0$. This enables the method of images to be used to solve for the stream function $\Psi(x, y, t)$, as the flow would be symmetric about $x=0$. The boundary conditions on the walls are now $u=0$ on $x= \pm 2 \beta$ and (2.10) on $y=1+\lambda$. The background component (3.1) and the unsteady component (3.2) are now modified by replacing the $\sin (n \pi x / \beta)$ term with $\sin (n \pi x /(2 \beta))$. This results in vorticity taking the form

$$
\omega=\sum_{m=1}^{\infty} \sum_{n=1}^{\infty} D_{m n} A_{m n}(t) \sin \left(\frac{m \pi y}{1+\lambda}\right) \sin \left(\frac{n \pi x}{2 \beta}\right),
$$

where

$$
D_{m n}=\left(\frac{n \pi}{2 \beta}\right)^{2}+\left(\frac{m \pi}{1+\lambda}\right)^{2}
$$

The perturbation density $\bar{\rho}$ takes the form

$$
\begin{aligned}
\bar{\rho}(x, y, t)=\sum_{m=1}^{\infty} & \sum_{n=1}^{\infty} B_{m n}(t) \sin \left(\frac{m \pi y}{1+\lambda}\right) \cos \left(\frac{n \pi x}{2 \beta}\right) \\
& +\sum_{m=1}^{\infty} B_{m 0}(t) \cos \left(\frac{(2 m-1) \pi y}{2(1+\lambda)}\right) .
\end{aligned}
$$

In the case of multiple drain holes located asymmetrically along the bottom of the tank, we consider two alternative drain hole configurations, with each drain hole having width $\alpha$. The first case (case 1) considers one drain hole centrally located and a second drain hole butted up against the right-hand wall. The second case (case 2) consists of a drain hole butted up against the right-hand wall and a second drain hole separated from it by a distance $\alpha$. The respective boundary conditions for the bottom 
of the tank for case 1 and case 2 are

$$
\begin{aligned}
& v(x, 0)=\left\{\begin{array}{cc}
-F & \text { if } x \in(-2 \beta,-2 \beta+\alpha), x \in(-\beta-\alpha / 2,-\beta+\alpha / 2), \\
& x \in(\beta-\alpha / 2, \beta+\alpha / 2) \text { or } x \in(2 \beta-\alpha, 2 \beta)
\end{array}\right. \\
& v(x, 0)=\left\{\begin{array}{cc}
-F & \text { if } x \in(-2 \beta,-2 \beta+\alpha), x \in(-2 \beta+2 \alpha,-2 \beta+3 \alpha), \\
0 & \begin{array}{c}
x \in(2 \beta-3 \alpha, 2 \beta-2 \alpha) \text { or } x \in(2 \beta-\alpha, 2 \beta)
\end{array}
\end{array}\right. \\
& \text { otherwise. }
\end{aligned}
$$

For case 1, the Fourier coefficients $B_{n}$ in the modified version of equation (3.1) have the form

$$
B_{n}=\frac{4 \beta F\left[\sin \left(\frac{n \pi(\alpha-2 \beta)}{2 \beta}\right)+\sin \left(\frac{n \pi(\alpha-2 \beta)}{4 \beta}\right)+\sin \left(\frac{n \pi(\alpha+2 \beta)}{4 \beta}\right)\right]}{n^{2} \pi^{2} \sinh \left[\frac{n \pi}{2 \beta}(1+\lambda)\right]},
$$

and for case 2,

$$
B_{n}=\frac{4 \beta F\left[\sin \left(\frac{n \pi(\alpha-2 \beta)}{2 \beta}\right)+\sin \left(\frac{n \pi(3 \alpha-2 \beta)}{2 \beta}\right)+\sin \left(\frac{n \pi(\beta-\alpha)}{\beta}\right)\right]}{n^{2} \pi^{2} \sinh \left[\frac{n \pi}{2 \beta}(1+\lambda)\right]} .
$$

The ordinary differential equations for the asymmetrically located drain holes are slightly different from those for one drain hole and two symmetrically located drain holes, as some of the basis functions are different from those in the previous derivations and the integration in $x$ is over the region $-2 \beta<x<2 \beta$. The method is the same as previously used, and thus for brevity we simply state the systems of ordinary differential equations. They are

$$
\begin{aligned}
A_{k l}^{\prime}(t)= & \frac{-1}{\beta(1+\lambda) D_{k l}} \int_{0}^{1+\lambda} \int_{-2 \beta}^{2 \beta} F(x, y, t) \sin \left(\frac{l \pi x}{2 \beta}\right) \sin \left(\frac{k \pi y}{1+\lambda}\right) d x d y \\
& \quad+\frac{l \pi}{2 \beta} \frac{B_{k l}(t)}{D_{k l}}-\mu D_{k l} A_{k l}(t), \quad k=1,2, \ldots, M, l=1,2, \ldots, N ; \\
B_{k 0}^{\prime}(t)= & \frac{-1}{2 \beta(1+\lambda)} \int_{0}^{1+\lambda} \int_{-2 \beta}^{2 \beta} G(x, y, t) \cos \left(\frac{(2 k-1) \pi y}{2(1+\lambda)}\right) d x d y \\
& -\gamma\left(\frac{(2 k-1) \pi}{2(1+\lambda)}\right)^{2} B_{k 0}(t), \quad k=1,2, \ldots, M ; \\
B_{k l}^{\prime}(t)= & \frac{-1}{\beta(1+\lambda)} \int_{0}^{1+\lambda} \int_{-2 \beta}^{2 \beta} G(x, y, t) \cos \left(\frac{l \pi x}{2 \beta}\right) \sin \left(\frac{k \pi y}{1+\lambda}\right) d x d y \\
& -\gamma D_{k l} B_{k l}(t), \quad k=1,2, \ldots, M, l=1,2, \ldots, N .
\end{aligned}
$$

The constants $D_{k l}$ are given by (3.12) and the functions $F(x, y, t)$ and $G(x, y, t)$ are defined as previously. 


\section{The linearized inviscid solution}

In this section, the linearized inviscid solution is presented and discussed. As the tank has two different density layers, there is an interface between the two fluids in the inviscid model. Initially this interface is a horizontal line defined by $y=1$. When the drain is opened, the interface begins to drop towards the drain hole and therefore changes with respect to the spatial variable $x$ and time variable $t$. Thus the interface can be defined as $y=\eta(x, t)$. The problem is highly nonlinear and therefore needs to be solved using numerical techniques. However, a linearized solution is possible for small Froude numbers $F$.

4.1. Single hole located symmetrically The derivation of the linearized interface function follows a similar technique to that used by Forbes and Hocking [4], and in the interest of conciseness will only be stated. For a single drain hole located centrally, the linearized interface function to order $F$ takes the form

$$
\eta(x, t)=1-t \frac{\alpha F}{\beta}-2 F \sum_{n=1}^{\infty} \frac{\sinh \left(\frac{n \pi \lambda}{\beta}\right) \sin \left(\frac{n \pi \alpha}{\beta}\right) \sin \left(t \sqrt{\frac{(1-D)}{T_{n}}}\right) \cos \left(\frac{n \pi x}{\beta}\right)}{n \pi \sqrt{\frac{(1-D)}{T_{n}}} \sinh \left[\frac{n \pi(1+\lambda)}{\beta}\right]},
$$

where

$$
T_{n}=\frac{D \operatorname{coth}\left(\frac{n \pi \lambda}{\beta}\right)+\operatorname{coth}\left(\frac{n \pi}{\beta}\right)}{\frac{n \pi}{\beta}} .
$$

4.2. Multiple holes located symmetrically The linearized interface solution $y=$ $\eta(x, t)$ for two symmetrically located drain holes is given by

$$
\eta(x, t)=1-t \frac{\alpha F}{\beta}-\sum_{n=1}^{\infty} \frac{B_{n}\left(\frac{n \pi}{\beta}\right) \sinh \left(\frac{n \pi \lambda}{\beta}\right)}{\sqrt{\frac{(1-D)}{T_{n}}}} \sin \left(t \sqrt{\frac{(1-D)}{T_{n}}}\right) \cos \left(\frac{n \pi x}{\beta}\right) .
$$

In this case, $B_{n}$ and $T_{n}$ are defined by (3.10) and (4.1), respectively.

4.3. Multiple holes located asymmetrically For multiple holes located asymmetrically, the linearized interfaces for both cases 1 and 2 have a similar form to that of equation (4.2), but with the $\beta$ terms in the infinite sum being replaced with $2 \beta$ to give

$$
\eta(x, t)=1-t \frac{\alpha F}{\beta}-\sum_{n=1}^{\infty} \frac{B_{n}\left(\frac{n \pi}{2 \beta}\right) \sinh \left(\frac{n \pi \lambda}{2 \beta}\right)}{\sqrt{\frac{(1-D)}{T_{n}}}} \sin \left(t \sqrt{\frac{(1-D)}{T_{n}}}\right) \cos \left(\frac{n \pi x}{2 \beta}\right)
$$

where $B_{n}$ is defined by (3.14) and (3.15) for cases 1 and 2, respectively. In both cases, $T_{n}$ takes the form

$$
T_{n}=\frac{D \operatorname{coth}\left(\frac{n \pi \lambda}{2 \beta}\right)+\operatorname{coth}\left(\frac{n \pi}{2 \beta}\right)}{\frac{n \pi}{2 \beta}}
$$


TABLE 1. The total outflow flux for each tank configuration with $F=0.03$ for half-drain widths $\alpha=3$ and $\alpha=4$.

\begin{tabular}{lcc}
\hline Configuration & $V_{\text {out }}$ for $\alpha=3$ & $V_{\text {out }}$ for $\alpha=4$ \\
\hline Single hole & 16.56 & 17.28 \\
Multiple holes, symmetric & 27.72 & 28.32 \\
Multiple holes, asymmetric, case 1 & 23.58 & 24.72 \\
Multiple holes, asymmetric, case 2 & 18.00 & 18.96 \\
\hline
\end{tabular}

\section{Presentation of results}

In this section, the results and findings of the problem are discussed, using the methods presented in the previous sections. Density and vorticity contour plots for tanks with various drain configurations, drain widths and flow rates are of particular interest. The time at which the upper, lighter fluid starts to withdraw from the tank is studied due to its applications in water management when withdrawing heavier polluted fluids from settling tanks. If the sum of the drain widths $2 \alpha$ and the Froude number $F$ are kept constant then, comparing such times at which the upper fluid starts to be withdrawn for each drain configuration, it can be concluded that the greater the time, the greater the outflow volume. In this paper we compare the outflow volume by using the dimensionless outflow flux for each tank configuration with $\alpha=3$ and $\alpha=4$ and the Froude number $F=0.03$. The approximate withdrawal times for each drain configuration for $\alpha=3$ are shown by density contour plots, but in the interest of conciseness the approximate time of withdrawal for tanks with $\alpha=4$ is only stated and used to determine the total outflow flux in Table 1 at the end of this section. The dimensionless total outflow flux of the lower, heavier fluid is $V_{\text {out }}$. The total outflow flux for a single drain hole and multiple drain holes located symmetrically is given by

$$
V_{\text {out }}=2 \alpha F t,
$$

and for multiple drain holes located asymmetrically by

$$
V_{\text {out }}=2 \alpha F t_{R}+\alpha F\left(t_{L}-t_{R}\right),
$$

where $t_{R}$ and $t_{L}$ are the times at which the upper fluid starts to be withdrawn from the right and left drain hole, respectively.

The viscosity and diffusion parameters are taken to be $\mu=\gamma=10^{-4}$, and $M=N=$ 25 Fourier coefficients are taken in each spatial variable unless specified otherwise. In particular, Figures 4(b), 12(a) and (b), and 14(a) and (b) use $M=N=31$ Fourier coefficients and were compared to $M=N=25$ Fourier coefficients for the same tank configurations to check the convergence of the numerical solutions. The dimensionless half-tank width and height of the upper fluid are $\beta=20$ and $\lambda=0.75$, respectively. Unless specified otherwise, the Froude number is $F=0.03$ and the density ratio is $D=0.97$. In the viscous solution, the interfacial region is distinguished by a smooth 
(a) Linearized interface evolution, $\alpha=2$

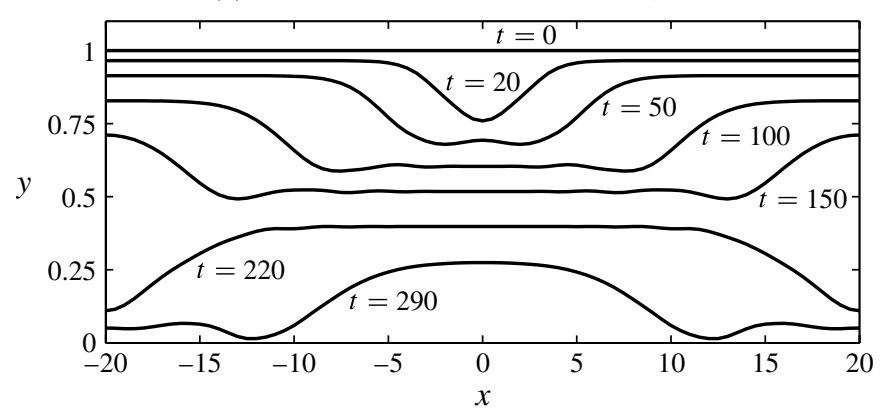

(b) Linearized interface evolution, $\alpha=4$

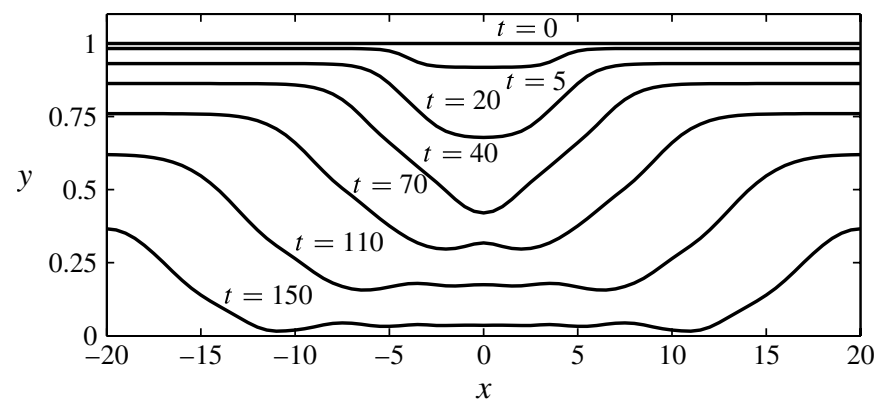

FIGURE 2. Evolution of the linearized interface for a tank with $\beta=20, \lambda=0.75, F=0.03$ and $D=0.97$, for (a) $\alpha=2$ at $t=0,20,50,100,150,220$ and 290; (b) $\alpha=4$ at $t=0,5,20,40,70,110$ and 150.

but rapid change in the density $\rho$, so that contours of this function are closely packed in this region. This is compared with the solution for the linearized interface discussed in Section 4.

5.1. Single hole located symmetrically When withdrawing fluid from the tank, it is assumed that the drain is impulsively turned on. It is expected that once the withdrawal process is started, the interface between the fluid regions would begin to drop towards the bottom of the tank, particularly in the region directly above the drain. This behaviour is shown for the linearized approximation in Figure 2(a) for half-drain width $\alpha=2$, where the evolution of the interface location is shown at seven different times. At $t=0$, the interface is horizontal as both fluids are at rest prior to the impulsive start of the withdrawal process. The profile at $t=20$ has a significant dip in the interface directly above the drain and remains horizontal across the rest of the tank. The profiles at $t=50,100$ and 150 show clearly that a travelling wave is produced due to the withdrawal and moves outward towards the walls of the tank. At the later time $t=220$, the waves have been reflected back towards the centre of the tank with a $180^{\circ}$ change of phase. These inward travelling waves have met in the centre of the tank by $t=290$ and further disturbances occur due to other outward travelling waves. 
(a) Linearized interface and density contours

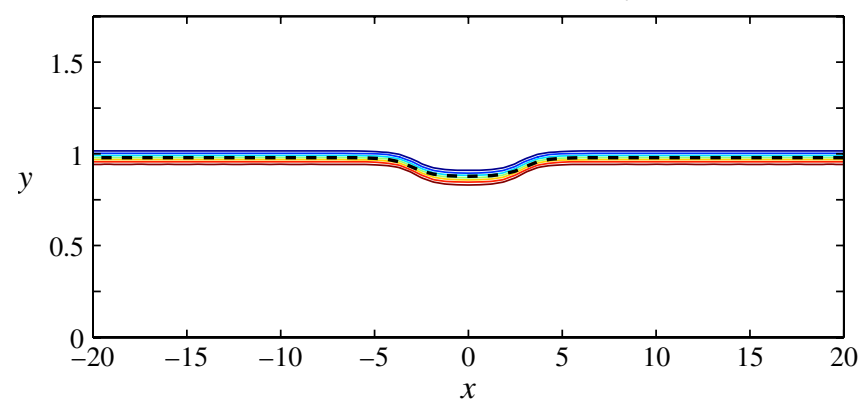

(b) Linearized interface and vorticity contours

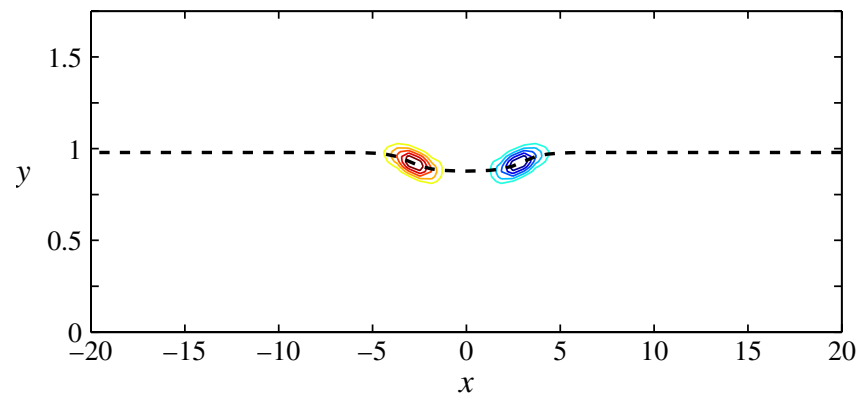

FIGURE 3. Linearized interface (dashed line) with contours of (a) density and (b) vorticity for a tank with $\alpha=3, \beta=20, \lambda=0.75, F=0.03$ and $D=0.97$ at $t=8$.

Figure 2(b) shows the evolution of the linearized interface for $\alpha=4$. In this case, the interface is drawn towards the drain at a much faster rate due to the wider drain, and as a consequence the outward travelling waves do not have time to reflect back off the tank walls before withdrawal is completed.

For a tank consisting of one drain hole, with $\alpha=3$, a typical density contour plot and vorticity contour plot for the fully viscous problem are shown in Figures 3(a) and (b), respectively, at time $t=8$, with a thick dashed line representing the inviscid linearized interface. The contours of the density $\rho(x, y, t)$ were computed by summing the density of the upper fluid $\rho_{1}$ and the perturbation density as defined by equation (3.6). In the density contour plots, the blank regions above and below the interface have density ranging from 0.970 to 0.973 and 0.997 to 1.000 , respectively, and the visible contours have values that increase in increments of 0.03 from 0.976 to 0.994 . The contours of the vorticity $\omega$ were computed using equation (3.4).

The initial conditions for the viscous solutions in Figure 3 were determined by assuming that the fluid is initially at rest and therefore that its velocity is zero. This is achieved by setting $A_{m n}(0)=0$ in equations (3.3) and (3.4). At $t=0$, the perturbation density function $\bar{\rho}$ is obtained from equation (3.6) and takes values $\bar{\rho}=0$ in the region $1<y<1+\lambda$ and $\bar{\rho}=1-D$ in the region $0<y<1$. So the initial values for the Fourier 
(a) Approximate viscous interface, linearized interface and density contours, $M=N=25$

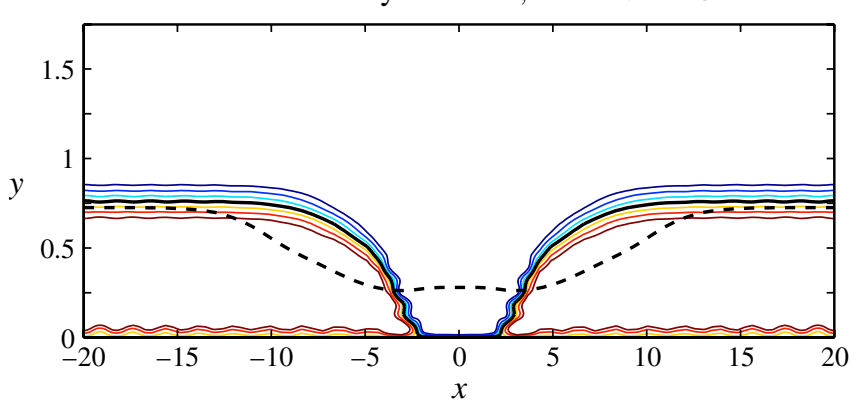

(b) Approximate viscous interface, linearized interface and density contours, $M=N=31$

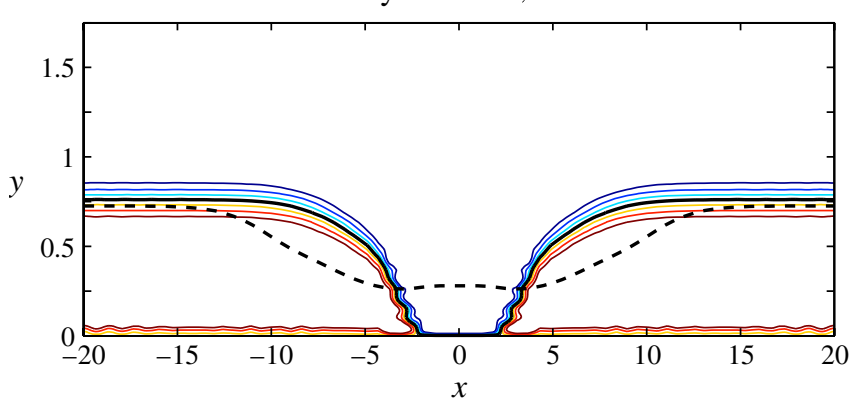

FIGURE 4. Linearized interface (dashed line), approximate viscous interface $\rho=0.985$ (thick line) and density contours with Fourier coefficients (a) $M=N=25$ and (b) $M=N=31$ for a tank with $\alpha=4$, $\beta=20, \lambda=0.75, F=0.03$ and $D=0.97$ at time $t=80$.

coefficients, $B_{m 0}(0)$ and $B_{m n}(0)$, are

$$
\begin{aligned}
& B_{m 0}(0)=\frac{4(1-D)}{(2 m-1) \pi} \sin \left(\frac{(2 m-1) \pi}{2(1+\lambda)}\right), \\
& B_{m n}(0)=0, \quad n \geq 1 .
\end{aligned}
$$

For the linearized solution, it was expected that the function $y=\eta(x, t)$ would be comparable with the fully nonlinear viscous solution in the region of the tank where the density contours are closely compacted together, showing a rapid change in density over a thin region of the tank for early times in the withdrawal process. This is confirmed in Figure 3(a), which shows a region of densely compacted contour lines in the middle of the diagram with the linearized interface lying directly in the middle of the nonlinear viscous interfacial region. From Figure 3(a), it is reasonable to assume that the fully viscous interface can be represented by the density contour that has the 
value midway between the density of the lighter and heavier fluids, in this case the density contour $\rho=0.985$.

Figure 3(b) shows the linearized interface overlaid on vorticity contours for the same parameters. The vorticity $\omega$ is essentially zero throughout the entire tank, suggesting that the flow is almost irrotational and confirming the approximate boundary condition (2.8). However, two small regions of relatively high vorticity are formed on the interface at precisely the points where the surface slopes are greatest. These regions of nonzero vorticity are antisymmetric in $x$ and range in absolute value from 0.009 on the outer contour to 0.045 on the innermost contour. They indicate that strongly rotational flow is possible at these locations, and they therefore have a significant influence on the physical flow behaviour. In a practical experiment, these are the regions in which turbulence might first be produced [10, p. 22].

As time increases, the linearized inviscid approximation becomes increasingly inaccurate. This can be seen in Figure 4, which shows the density contours for the fully nonlinear viscous problem combined with the linearized inviscid interface at time $t=80$. In particular, the nonlinear interface has drawn down into the sink, whereas for the linearized solution this has not yet occurred. This suggests that nonlinear and viscous effects are increasing the speed at which the upper fluid is being pulled towards the drain. As an indicator of the mean location of the viscous "interface", the intermediate density contour $\rho=0.985$ has been selected, and is shown with a thick black line in Figure 4. Figures 4(a) and (b) were generated using $M=N=25$ and $M=N=31$ Fourier coefficients, respectively. Figure 4(b) shows little change compared to Figure 4(a), which suggests that using any more than $M=N=25$ Fourier coefficients has negligible impact on the numerical solution and implies that the Fourier coefficients have converged. Indeed, a careful examination of the Fourier coefficients $A_{m n}$ and $B_{m n}$ at selected times confirms that they are insignificant beyond about order 30 .

Figure 5(a) shows the density contours for a tank with half-drain width $\alpha=3$ and density ratio $D=0.97$ at time $t=92$. The thick black line is the density contour $\rho=0.985$ and is therefore an indicator of the viscous interface. At time $t=92$, this contour touches the bottom of the tank. From this time onwards there is continual withdrawal of the lighter fluid from the tank, and thus the density contours, which are an indication of the true interface between the fluids, become increasingly steep as time goes on, to the point at which the density contours turn in under themselves and separate into two distinct regions on the left and right of the drain hole, encapsulating the denser fluid. These two regions of high density fluid are separated by a funnel of lighter fluid approximately two-thirds of the drain width. So as well as the lighter fluid being drained out, the heavier fluid is also being extracted, in a process of viscous entrainment.

Figure 5(b) shows that at $t=92$ there are also two symmetrically located regions of vorticity with high rotation just above the inside edges of the drain hole. These regions of nonzero vorticity have absolute contour values ranging from 0.16 to 0.83 and align themselves with the shape of the funnel which separates the two regions of fluid at 
(a) Approximate viscous interface and density contours

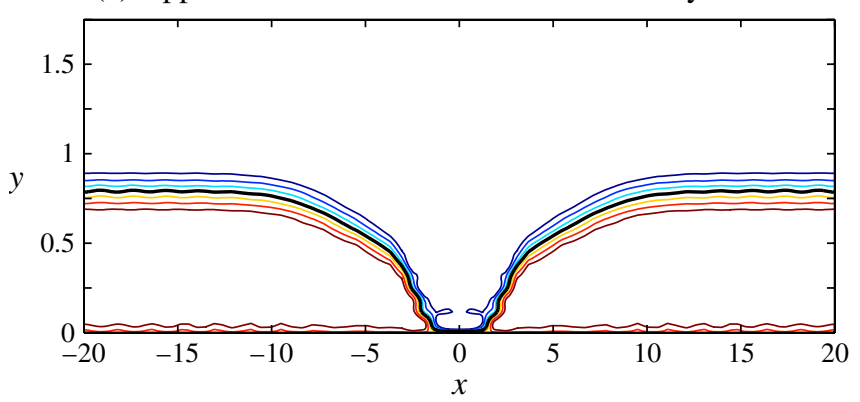

(b) Approximate viscous interface and vorticity contours

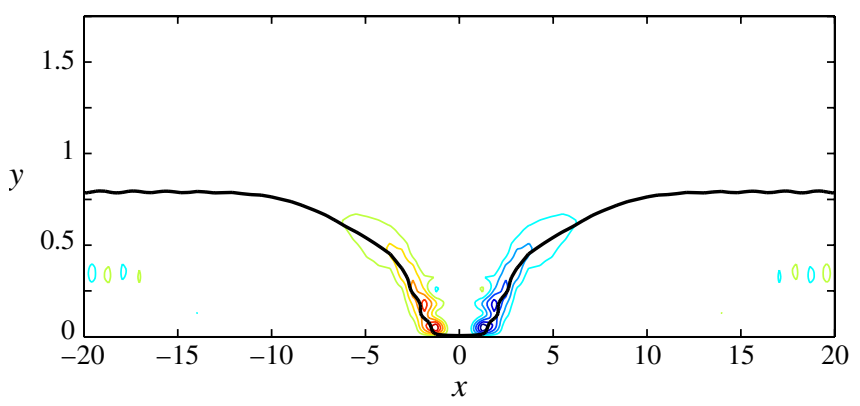

FIGURE 5. Approximate viscous interface $\rho=0.985$ (thick line) with contours of (a) density and (b) vorticity for a tank with $\alpha=3, \beta=20, \lambda=0.75, F=0.03$ and $D=0.97$ at $t=92$.

this time. This is to be expected, since the regions of relatively high vorticity occur at points in which the density contour $\rho=0.985$ and thus the viscous interface are at their steepest. These regions of vorticity create strongly rotational flow, leading to mixing of the two fluids through viscous entrainment. The denser fluid ultimately separates into two isolated regions either side of a jet of the lighter fluid. Similar results are achieved for varying drain widths and flow rates, although the times at which such results occur depend on these parameters.

Figure 6 gives a graphical view of the approximate time to withdrawal for different values of the half-drain width $\alpha$ and Froude number $F$. If the Froude number $F$ is increased and the half-drain width $\alpha$ is kept the same, then the time of initial withdrawal of the less dense fluid decreases. If $\alpha$ is increased and $F$ is kept the same, then the time of initial withdrawal of the upper fluid decreases. For example, if $F=0.03$ and $\alpha$ is increased to 4 , then the approximate time to withdrawal drops to $t=72$. This time value is used to determine the total outflow flux in Table 1 at the end of this section, for a single symmetrically located drain hole. To illustrate these findings, Figure 7 shows density contours for a tank with $\alpha=5$ and $F=0.06$. The time of initial withdrawal of the upper fluid is $t=27$ and the figure shows the approximate viscous interface spanning more than half the drain at this time. 


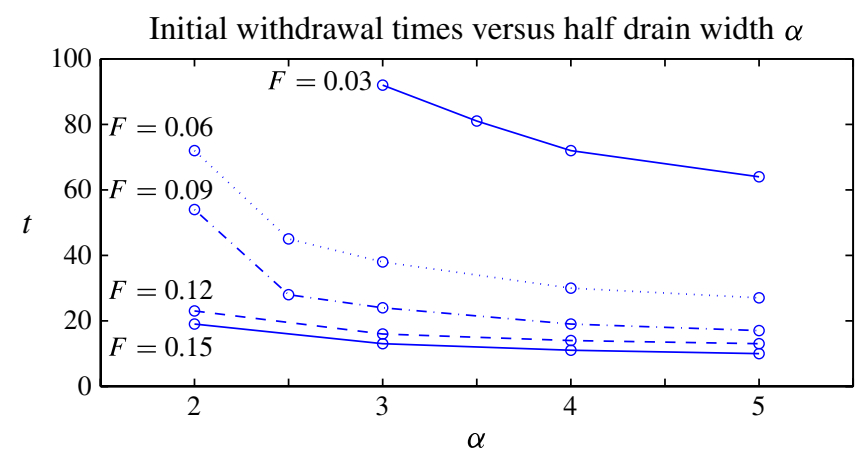

FIGURE 6. Approximate initial withdrawal times for tanks with $\alpha=2,2.5,3,3.5,4$ and 5; Froude numbers $F=0.03,0.06,0.09,0.12$ and $0.15 ; \beta=20 ; \lambda=0.75$ and $D=0.97$.

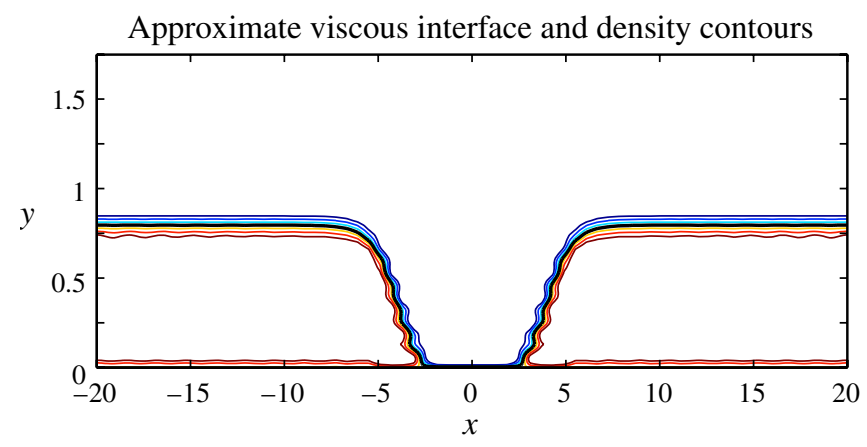

FIGURE 7. Approximate viscous interface $\rho=0.985$ (thick line) and density contours for a tank with $\alpha=5$, $\beta=20, \lambda=0.75, F=0.06$ and $D=0.97$ at time $t=27$.

5.2. Multiple holes located symmetrically For a tank with two symmetrically located drain holes, equations (3.6) and (3.4) are again used to compute the density $\rho(x, y, t)$ and vorticity $\omega$ contour diagrams, respectively. The initial conditions are the same as for a single hole located symmetrically and the linearized interface is computed using (4.2).

Figure 8(a) displays the linearized interface overlaid with density contours. It confirms the findings in the previous section, in that the linearized interface function shown as the thick black dashed line is a reliable estimate for the fully nonlinear viscous interface for early times in the withdrawal process, as it lies directly in the middle of the viscous interfacial region. Figure 8(b) shows the same interface, but now combined with vorticity contours. There are four elliptical contour regions consisting of two pairs symmetrically located about $x=0$, clustered at locations along the linearized interface. This reaffirms that the vorticity has its greatest absolute value at points where the interface is steepest. In this case, the absolute vorticity contour values increase from 0.006 to 0.030 with the largest values lying directly on the 
(a) Linearized interface and density contours

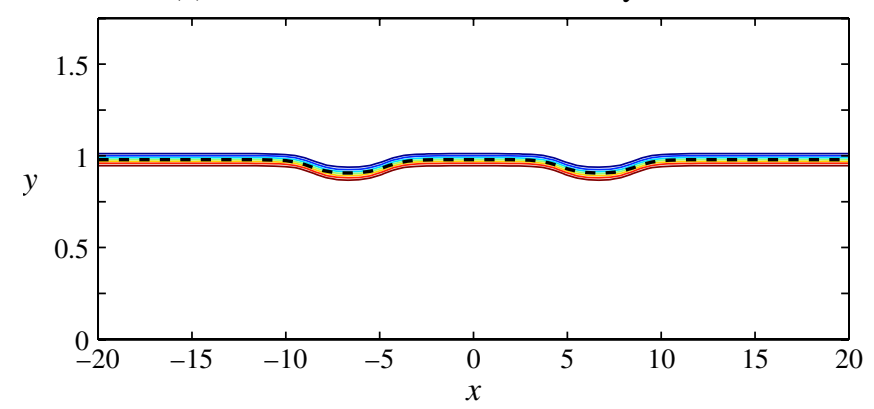

(b) Linearized interface and vorticity contours

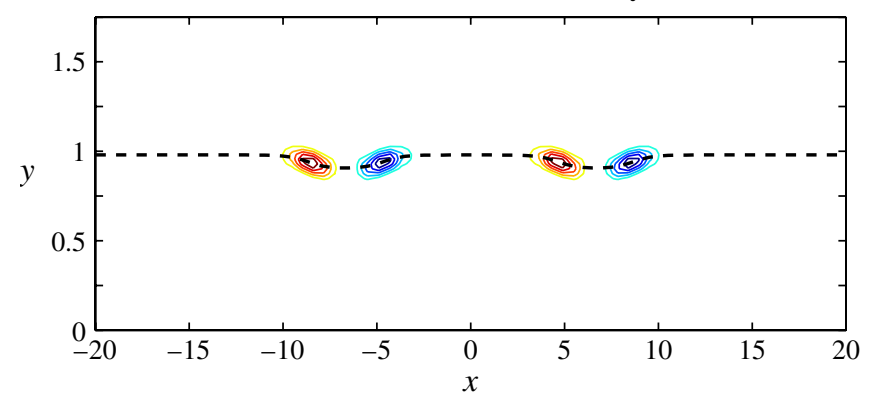

FIGURE 8. Linearized interface (dashed line) with contours of (a) density and (b) vorticity for a tank with $\alpha=4, \beta=20, \lambda=0.75, F=0.03$ and $D=0.97$ at $t=6$.

linearized interface. Like the single drain hole configuration, away from the interfacial region, vorticity is essentially zero.

For a tank configuration of two symmetrically located drain holes with $\alpha=3$, the time at which the lighter fluid starts to withdraw from the tank is $t=154$. This is illustrated in Figure 9(a), which shows the viscous interface, taken to be approximated by the contour $\rho=0.985$, reaching the bottom of the tank and spanning most of each drain hole. As with the single drain hole case, a wedge type funnel formation appears above each drain hole, forming four distinct regions in the tank, one of light fluid above the wedge and three encased by the wedge, sides and bottom of the tank. The lighter fluid is draining out over the central two thirds of each drain. This process is similar to the single drain hole case. At the sides of the drains there is heavier fluid exiting, due to the high vorticity on the sides of the drains and the process of viscous entrainment. The vorticity contours in this case range in value from 0.14 to 0.73 . Due to the high vorticity on the boundaries of the wedge, shown in Figure 9(b), the less dense fluid is being spread along the bottom of the tank and mixing with the heavier fluid, forming the three regions of heavier fluid that are enclosed by the interface. The central heavier region has less dense fluid on the outer edges and becomes denser towards the centre. The outer two dense regions exhibit similar behaviour with the additional localized 
(a) Approximate viscous interface and density contours

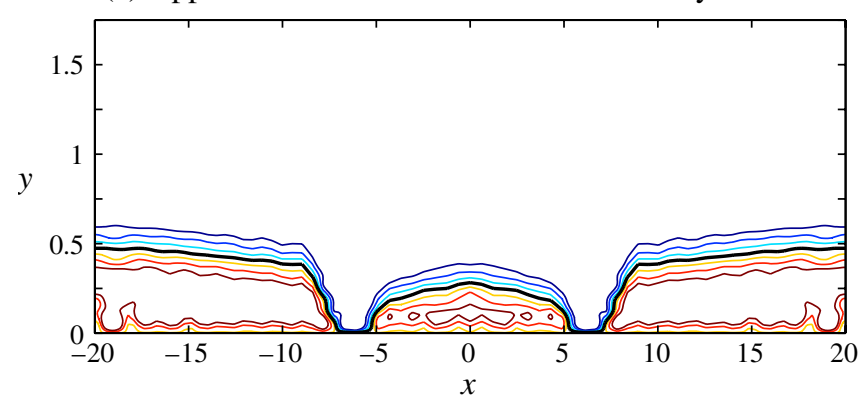

(b) Approximate viscous interface and vorticity contours

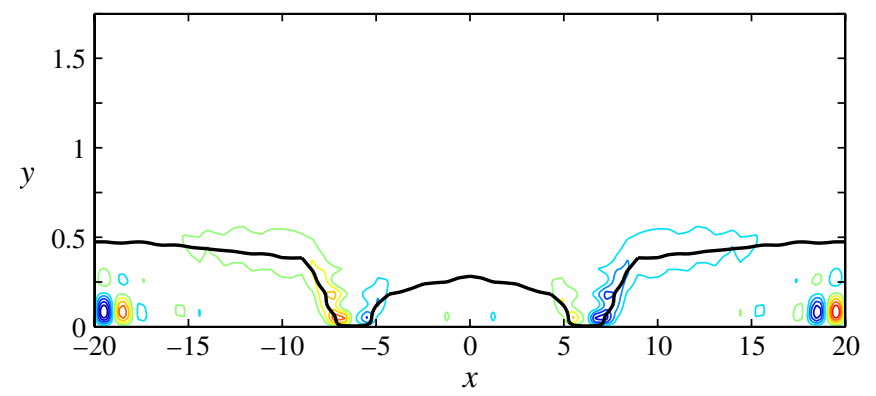

Figure 9. Approximate viscous interface $\rho=0.985$ (thick line) with contours of (a) density and (b) vorticity for a tank with $\alpha=3, \beta=20, \lambda=0.75, F=0.03$ and $D=0.97$ at $t=154$.

high density regions in the corners of the tank. This again is due to the high vorticity that develops in the corners of the tank.

When varying the dimensionless half-drain width $\alpha$ and keeping all other parameters the same, the results are similar. The only differences occur in the times at which the lighter fluid begins to exit the tank. For a tank with increased drain widths, for example $\alpha=4$, the time of withdrawal occurs at approximately $t=118$; this is the time used to calculate the outflow flux value in Table 1. For decreased drain widths, for example $\alpha=2$, the time to withdrawal occurs at approximately $t=224$. If the Froude number $F$ is increased, then the time of initial withdrawal of the less dense fluid decreases and vice versa. The density ratio has the opposite effect, that is, when the density ratio is less than 0.97 , the time of initial withdrawal of the upper fluid is increased.

5.3. Multiple holes located asymmetrically In the case of two asymmetrically located drain holes, equations (3.13) and (3.11) are used to produce the density $\rho(x, y, t)$ and vorticity $\omega$ contour diagrams, respectively. The initial conditions are the same as previous drain hole configurations and the linearized interface function is calculated using (4.3) together with (3.14) for case 1 and (3.15) for case 2. 


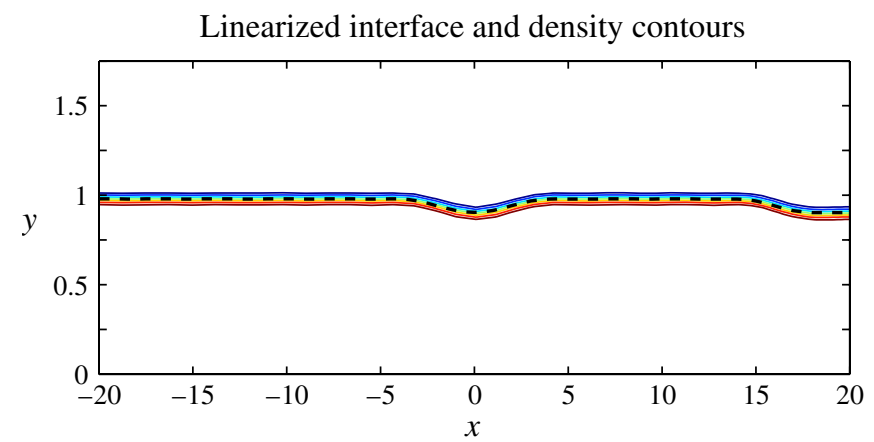

FIGURE 10. Case 1: linearized interface (dashed line) overlaid with density contours for a tank with $\alpha=4$, $\beta=20, \lambda=0.75, F=0.03$ and $D=0.97$ at $t=6$.

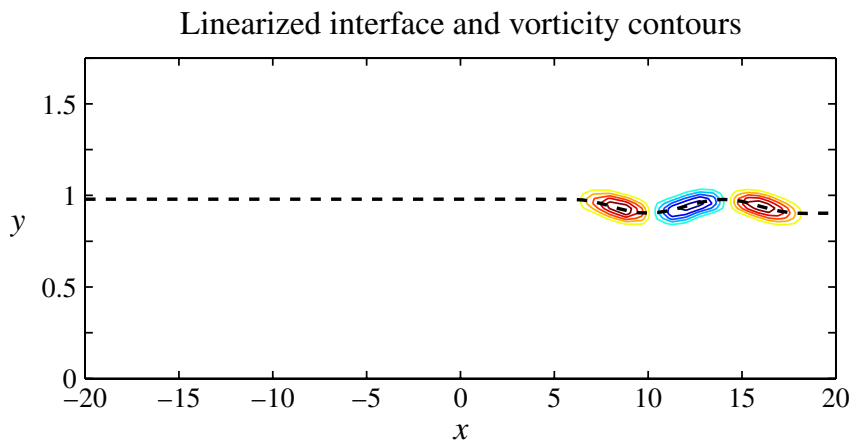

FIGURE 11. Case 2: linearized interface (dashed line) overlaid with vorticity contours for a tank with $\alpha=4$, $\beta=20, \lambda=0.75, F=0.03$ and $D=0.97$ at $t=6$.

Figure 10 shows the linearized interface function for case 1 combined with density contour plots, and Figure 11 shows the linearized interface function for case 2 combined with vorticity contour plots. Both figures give further evidence of the reliability of the linearized interface function as an accurate indicator of the viscous interface at early times in the withdrawal process and that vorticity is greatest at locations on the viscous interface where the interface changes most rapidly.

Figure 12(a) shows a tank with configuration as defined by case 1 , with $\alpha=3$. In this configuration, it was found that at time $t=90$ the approximate viscous interface shown as the thick black line spans the right two thirds of the drain and thus the lighter, upper fluid starts to be withdrawn out of the tank through this drain hole and produces the now familiar wedge formation along the viscous interface. This withdrawal time is in agreement with what was found in the single drain hole case and is to be expected, due to the fact that the central drain hole has little influence on the fluid particles above the right-hand wall and can therefore be considered as the left side of the single drain 
(a) Viscous interface and density contours, $t=90$

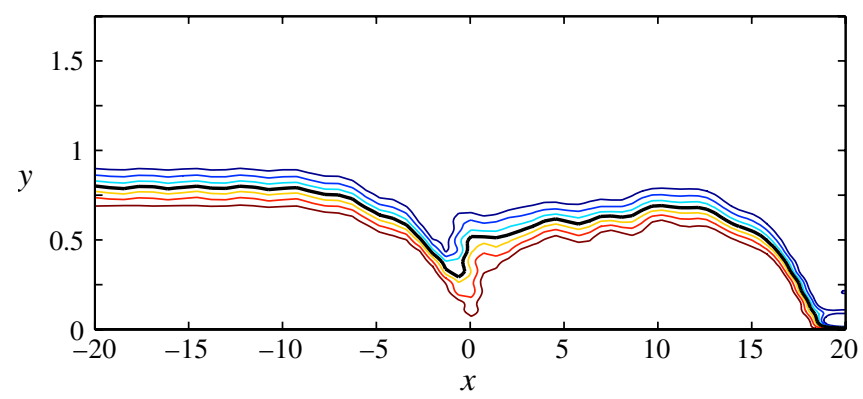

(b) Viscous interface and density contours, $t=172$

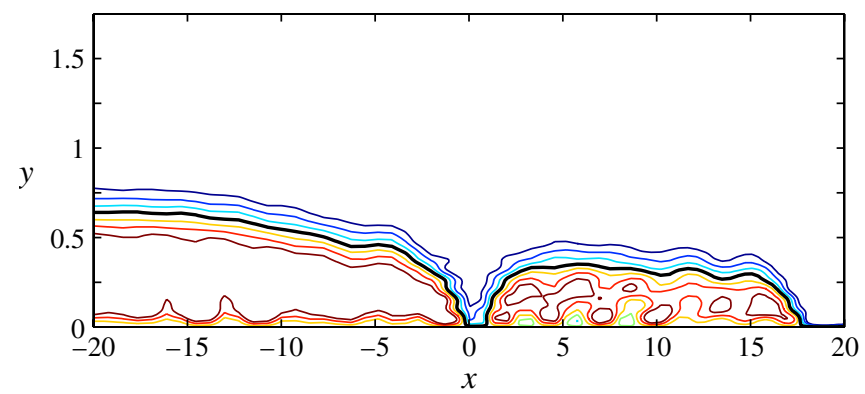

FigURE 12. Case 1: approximate viscous interface $\rho=0.985$ (thick line) and density contours for a tank with $\alpha=3, \beta=20, \lambda=0.75, F=0.03$ and $D=0.97$. Results are shown at (a) $t=90$ and (b) $t=172$.

hole tank. For times $t>90$, the wedge formation remains and there is a continual withdrawal of the less dense fluid across the right two thirds of the drain.

Figure 12(b) shows the same tank but at the later time $t=172$. This is the time at which the less dense fluid starts to be withdrawn from the central drain hole. At this time, a more pronounced wedge in the contours has formed, isolating three distinct regions of fluid, one of less dense fluid above the interface and two regions of heavier fluid. There is a considerable drop in heavier fluid volume being withdrawn from this central hole compared to previous cases, as the withdrawal of fluid only occurs across one third of the drain, due to high vorticity near the sides of the drains. This behaviour is consistent with other values of $\alpha$. When $\alpha=4$ and all other parameters are kept the same, the time of approximate initial withdrawal through the right drain hole is $t=72$, and at $t=134$ the upper fluid starts to withdraw from the left drain hole. These values are used to determine the outflow flux in Table 1 . The vorticity again has its absolute maximum along the contour approximation to the viscous interface; this is not shown here in the interest of brevity. Similar outcomes occur at other values of the parameters $\alpha, F$ and $D$.

Figure 13 shows the approximate viscous interface as defined by the density contour $\rho=0.985$ overlaid on stream function contours. The stream function contours were 


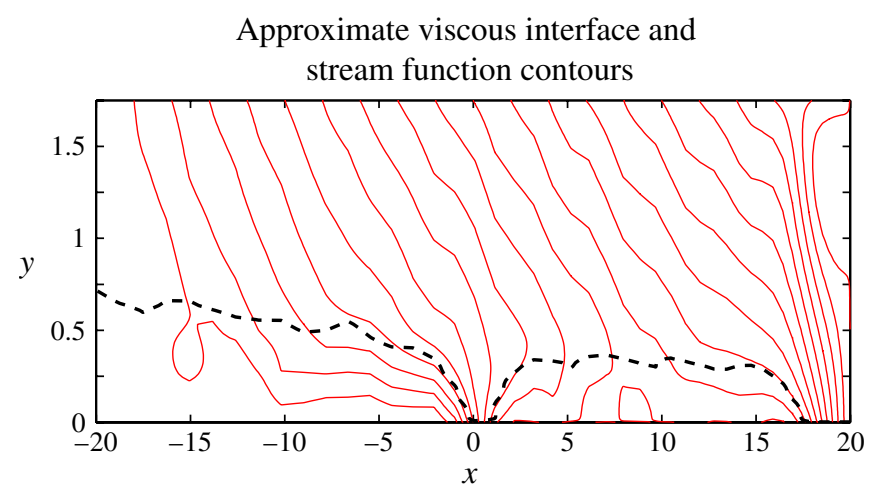

FIGURE 13. Approximate viscous interface $\rho=0.985$ (dashed line) and stream function contours for a tank with $\alpha=3, \beta=20, \lambda=0.75, F=0.03$ and $D=0.97$ at $t=172$.

computed using the modified form of equation (3.3). The loop in the bottom left corner is not significant, since $\Psi \approx 0$ in that corner. The streamlines give further evidence that the density contour $\rho=0.985$ is a good approximation to the viscous interface as the interface is tangential to streamlines near both drains.

For case 2 of asymmetrically located drain holes, it was found that the time in which the less dense fluid begins to withdraw out of the right drain is $t=78$, and it occurs over the right two thirds of the drain. At this time the heavier lower fluid is still being withdrawn from the left drain. At time $t=122$ the upper fluid begins to be withdrawn from the left drain. From this time onwards there is a continual outflow of the upper fluid across the entire right drain and the right two thirds of the left drain. The density contour plots combined with the approximate viscous interface are shown in Figures 14(a) and (b), respectively. For $\alpha=4$, the respective approximate withdrawal times out through the right and left drain holes are $t=62$ and $t=96$, and are used to calculate the total outflow flux in Table 1 .

Figures 12 and 14 were produced using $M=N=31$ Fourier coefficients and were compared to the same plots when $M=N=25$ Fourier coefficients are used. Varying the number of Fourier coefficients does not influence the withdrawal times and thus the results are unchanged, but it does reduce the wave peaks on the density contours marginally, in the same way as was shown when comparing Figures 4(a) and (b). In the interest of brevity, the plots using a truncation with $M=N=25$ are not shown here, but this comparison confirms the convergence of the numerical results.

Table 1 gives a summary of the total outflow flux for all tank configurations with $F=0.03$ for $\alpha=3$ and 4 . The total outflow flux was computed using equations (5.1) and (5.2). In the tank configurations discussed above with $\alpha=3$ and $\alpha=4$, the outflow flux and thus the withdrawal volume are greatest when the drain holes are symmetrically placed along the line of withdrawal, followed by the asymmetric case 1 . 
(a) Viscous interface and density contours, $t=78$

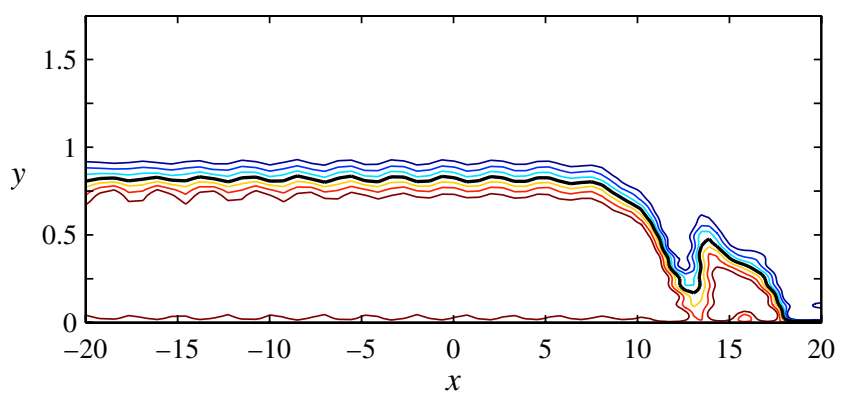

(b) Viscous interface and density contours, $t=122$

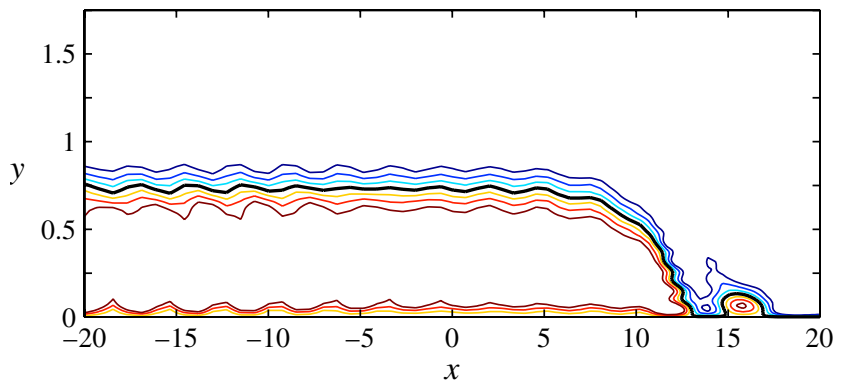

FigURE 14. Case 2: approximate viscous interface $\rho=0.985$ (thick line) and density contours for a tank with $\alpha=3, \beta=20, \lambda=0.75, F=0.03$ and $D=0.97$. Results are shown at (a) $t=78$ and (b) $t=122$.

\section{Conclusions and discussion}

This paper has focused on withdrawing fluid from a rectangular tank containing a two-layered viscous fluid. The tanks themselves were designed with single and multiple drain holes, not necessarily symmetrically placed. The aim was to achieve maximum withdrawal volume of the denser fluid before the viscous interface reaches the sink or sinks. It was observed that the linearized inviscid interface lay directly in the centre of the nonlinear viscous interfacial region at early times in the withdrawal process, and it was therefore concluded that the approximate viscous interface could be taken to be the density contour $\rho=0.985$. In this paper, we have taken the withdrawal time to be when the contour $\rho=0.985$ first makes contact with the drain. However, there is some arbitrariness in this definition, which could lead to different times of withdrawal. This was also encountered by Stokes et al. [11] (see in particular their Figure 5).

It was found that, as the outflow rate and drain width increased, the time at which the less dense fluid started to be withdrawn from the tank decreased. This withdrawal time increased as the density ratio between the two fluids was decreased. For tanks with two symmetrically located drain holes, the depressions in the density contours were much less pronounced than for a single drain hole located symmetrically and for 
multiple drain holes located asymmetrically. Also, the time at which the less dense fluid starts to withdraw from the multiple symmetrically located drain hole tank far exceeds the times for the other tank configurations and thus the volume of lower fluid being withdrawn is maximized. This implies that the outflow of heavier fluid is much smoother and more efficient for a tank with two symmetrically located drain holes compared with other tank configurations. Therefore, in situations where the heavier fluid is being selectively withdrawn, it is best to have multiple drain holes that are evenly spaced along the line of withdrawal. This is because wave formation at the interface is suppressed, so delaying the time at which the interface reaches the drain holes.

Like in the work of Forbes and Hocking [4], the linearized interface between the two fluids coincides with the nonlinear interfacial region for early times in the withdrawal process, until nonlinear and viscous effects take over. Nevertheless, the curvature singularities encountered by Forbes and Hocking [4] for wide drains were not seen here, and this may be a consequence of the viscous effects, and the vorticity they create.

It was anticipated that the interface between the fluids might roll up on itself and create a reverse mushroom type formation as found by Forbes [3]. However, this formation did not eventuate in the cases considered in this study. It is, however, still possible that such effects may be produced at different parameter values or sink geometries, but this awaits further work.

\section{Acknowledgements}

The work of the first author was supported by a Tasmania Graduate Research Scholarship. Assistance from Anita Griffin with proofreading and editing is gratefully acknowledged.

\section{References}

[1] M. Ahmed, I. Hassan and N. Esmail, "The onset of gas pull-through during dual discharge from a stratified two-phase region: theoretical analysis", Phys. Fluids 16 (2004) 3385-3392; doi:10.1063/1.1771619.

[2] D. E. Farrow and G. C. Hocking, "A numerical model for withdrawal from a two-layer fluid", J. Fluid Mech. 549 (2006) 141-157; doi:10.1017/S0022112005007561.

[3] L. K. Forbes, "The Rayleigh-Taylor instability for inviscid and viscous fluids", J. Engrg. Math. 65 (2009) 273-290; doi:10.1007/s10665-009-9288-9.

[4] L. K. Forbes and G. C. Hocking, "Unsteady draining flows from a rectangular tank", Phys. Fluids 19 (2007) 082104; doi:10.1063/1.2759891.

[5] G. C. Hocking, "Critical withdrawal from a two-layer fluid through a line sink", J. Engrg. Math. 25 (1991) 1-11; doi:10.1007/BF00036598.

[6] G. C. Hocking and H. Zhang, "A note on withdrawal from a two-layer fluid through a line sink in a porous medium”, ANZIAM J. 50 (2008) 101-110; doi:10.1017/S144618110800028X.

[7] J. Imberger and P. F. Hamblin, "Dynamics of lakes, reservoirs and cooling ponds", Annu. Rev. Fluid Mech. 14 (1982) 153-187; doi:10.1146/annurev.fl.14.010182.001101.

[8] J. Imberger, R. Thompson and C. Fandry, "Selective withdrawal from a finite rectangular tank", J. Fluid Mech. 78 (1976) 489-512; doi:10.1017/S0022112076002577. 
[9] J. R. Lister, "Selective withdrawal from a viscous two-layer system", J. Fluid Mech. 198 (1989) 231-254; doi:10.1017/S002211208900011X.

[10] S. B. Pope, Turbulent flows (Cambridge University Press, Cambridge, 2000).

[11] T. E. Stokes, G. C. Hocking and L. K. Forbes, "Unsteady flow induced by a withdrawal point beneath a free surface", ANZIAM J. 47 (2005) 185-202; doi:10.1017/S1446181100009986.

[12] I. R. Wood, "Selective withdrawal from a stably stratified fluid", J. Fluid Mech. 32 (1968) 209-223; doi:10.1017/S0022112068000686. 